\title{
Telemedicina en tiempos de pandemia
}

\author{
Telemedicine in times of pandemic
}

\author{
Alejandro I Trejo Castro, * Diego Carrión Álvarez, ${ }^{\ddagger}$ Julio César Salas Alanís ${ }^{\ddagger}$ \\ Citar como: Trejo CAI, Carrión ÁD, Salas AJC. Telemedicina en tiempos de pandemia. Acta Med \\ Grupo Angeles. 2021; 19 (s1): s80-s83. https://dx.doi.org/10.35366/101034
}

\section{Resumen}

El propósito de este trabajo es analizar las ventajas y desventajas de la telemedicina durante la pandemia. Se realizó una revisión sistemática en Web of Science con las palabras clave "COVID-19 and Dermatology and Telemedicine" y "Telemedicine and Dermatology and Pandemic", arrojando 11 artículos, de los cuales nueve fueron analizados para esta revisión. Se encontró que algunos estudios reportan que más de $70 \%$ de las consultas físicas pudieron haber sido sustituidas por la telemedicina; además de que aún existe una resistencia a este tipo de consultas por los pacientes (Datos Wellmedic). La telemedicina es una herramienta que posee ventajas importantes durante tiempos de crisis, disminuyendo el riesgo de exposición de pacientes y médicos, así como una mejor distribución del uso de equipo personal de protección. Sin embargo, posee limitaciones en países en vías de desarrollo o poblaciones rurales por la falta de equipo y la resistencia de pacientes y médicos a utilizarla, además la carencia de un marco legal es clave en algunos países. Sin embargo, el costo-beneficio se considera mayor. Este es el primer artículo que habla acerca del impacto de la telemedicina durante la pandemia en México.

Palabras clave: Telemedicina, dermatología, COVID-19, pandemia.

\section{INTRODUCCIÓN}

La telemedicina ha estado presente durante algunos milenios, pues se considera que la comunicación en papiros y tablillas acerca de enfermedades en la antigua Grecia, Roma y Egipto marcan precursores de la telemedicina del día de hoy. ${ }^{1}$ El término oficial fue definido como "entrega de servicios de salud vía telecomunicaciones, incluyendo

\section{Abstract}

The purpose of this article is to analyse the advantages and disadvantages in telemedicine during the pandemic. We conducted a systematic review in Web of Science with the keywords "COVID-19 and Dermatology and Telemedicine" and "Telemedicine and Dermatology and Pandemic". From 11 articles we included nine. We found in some papers that more than $70 \%$ of the physical consultation could be substituted by telemedicine, as well as the resistance from patients in the use of such tool (WellMedic). Telemedicine is a tool that possesses relevant advantages in times of crisis, reducing the risk of exposition for patients and health providers and better distribution of the personal protection equipment. However, it has technical limitations in developing countries or rural areas, as well as the resistance from patients and doctors to use it. Furthermore, there is a lack of legal regulations in some countries. However, the cost-benefit has proven to be superior. This is the first article that talks about telemedicine in Mexico during this pandemic and its impact.

Keywords: Telemedicine, dermatology, COVID-19, pandemic.

consulta interactiva y servicios diagnósticos" en 1993 por la National Library of Medical Subject Headings. ${ }^{2}$ Aunque el primer ejemplo formal de esta práctica data de 1920, cuando médicos en la costa utilizaban comunicación de radio de onda corta para comunicar información médica con sus homólogos en los barcos. ${ }^{3}$ Conforme la tecnología fue avanzando, también las aplicaciones en telemedicina, permitiéndonos hoy en día el uso de cámaras y pantallas

* Escuela de Ingeniería y Ciencias, Tecnológico de Monterrey, Monterrey, Nuevo León, México.

‡ Dystrophic Epidermolysis Bullosa Research Association, Guadalupe, Correspondencia: Julio César Salas Alanís Nuevo León, México.

Correo electrónico: drjuliosalas@gmail.com

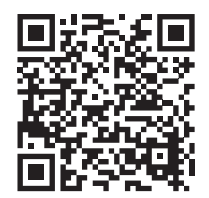


de alta definición, comunicación en tiempo real y uso de la nube en un contexto clínico multidisciplinario, ${ }^{4}$ así como el crecimiento exponencial de la industria de la telemedicina en los últimos años, con una proyección de mercado esperada de más de 130 billones de dólares para el año 2025. ${ }^{5}$ Sus modelos incluyen el desarrollo de programas directos a los consumidores sin la necesidad de un hospital o clínica interviniendo en el proceso; este sector también ha crecido de manera importante. ${ }^{6}$

La telemedicina es una herramienta que facilita la consulta con especialistas, incrementando su uso en especialidades como Neurología, ${ }^{7}$ Dermatología ${ }^{8}$ o Traumatología $;{ }^{9}$ incluso se ha replanteado su uso como herramienta de aprendizaje a distancia para médicos en formación. ${ }^{10}$ Sin embargo, no está libre de desafíos, entre ellos las regulaciones legales alrededor de su uso, ${ }^{11}$ la falta de tecnología en poblaciones rurales que podrían beneficiarse de manera importante ${ }^{12}$ y la polémica detrás de cómo afecta las relaciones médico-paciente. ${ }^{13}$

Con la llegada de la pandemia, esta práctica se ha vuelto más común, algunos artículos reportan un incremento de hasta $683 \%,{ }^{14}$ el cual se ha visto con mayor demanda en algunas especialidades como la dermatología para el seguimiento y diagnóstico. ${ }^{15}$ El objetivo de esta revisión es analizar la importancia, el uso y las ventajas de este instrumento de comunicación.

\section{MATERIAL Y MÉTODOS}

Se realizó una búsqueda sistemática en Web of Science con las palabras clave "Telemedicine and Dermatology and Pandemic" y "Telemedicine and COVID-19 and Dermatology" incluyendo todos los artículos disponibles de acuerdo con la metodología PRISMA. Analizamos los datos provistos por los artículos. La búsqueda incluye trabajos de investigación indexados en la base de datos hasta el 14 de noviembre de 2020.

\section{RESULTADOS}

De los artículos incluidos con las palabras clave se encontraron 11 trabajos de investigación, de los cuales se incluyeron nueve. Los otros dos artículos fueron excluidos por confusión en los datos. Seis de éstos fueron publicados en Estados Unidos. Cuatro proponen flujogramas, algoritmos o metodologías para mejorar la atención. Tres artículos corresponden a investigaciones originales, con cuatro cartas al editor y dos puntos de vista. Los detalles de seis de las publicaciones se encuentran en la Tabla 1.

Dentro de los artículos originales analizados, se encontró que $71.2 \%$ de los pacientes que acudieron a consulta física pudieron haber sido manejados por teledermatología; de

Tabla 1: Revistas incluidas en la revisión sistemática.

\begin{tabular}{|c|c|c|c|c|c|}
\hline Título & $\begin{array}{l}\text { Fecha de } \\
\text { publicación }\end{array}$ & $\begin{array}{l}\text { Tipo de } \\
\text { artículo }\end{array}$ & $\begin{array}{l}\text { País de } \\
\text { origen }\end{array}$ & $\begin{array}{l}\text { Sugerencia } \\
\text { de método o } \\
\text { algoritmo }\end{array}$ & Referencia \\
\hline $\begin{array}{l}\text { - Changing paradigms of dermatology } \\
\text { practice in developing nations in the } \\
\text { shadow of COVID-19: lessons learnt } \\
\text { from the pandemic }\end{array}$ & $\begin{array}{l}\text { Julio } \\
2020\end{array}$ & $\begin{array}{l}\text { Artículo } \\
\text { breve }\end{array}$ & India & No & $\begin{array}{l}\text { Kumar et al. } \\
2020\end{array}$ \\
\hline $\begin{array}{l}\text { Conducting inpatient dermatology } \\
\text { consultations and maintaining } \\
\text { resident education in the COVID-19 } \\
\text { telemedicine era }\end{array}$ & $\begin{array}{l}\text { Octubre } \\
2020\end{array}$ & $\begin{array}{l}\text { Carta al } \\
\text { editor }\end{array}$ & $\begin{array}{l}\text { Estados } \\
\text { Unidos }\end{array}$ & Sí & $\begin{array}{l}\text { Hammond } \\
\text { et al. } 2020\end{array}$ \\
\hline $\begin{array}{l}\text { - Growing role of telemedicine in } \\
\text { dermatology: a practical, timely } \\
\text { application for skin cancer screening } \\
\text { in organ transplant recipients }\end{array}$ & $\begin{array}{c}\text { Septiembre } \\
2020\end{array}$ & $\begin{array}{l}\text { Carta al } \\
\text { editor }\end{array}$ & Canadá & Sí & $\begin{array}{l}\text { Abi Rafeh et al. } \\
2020\end{array}$ \\
\hline $\begin{array}{l}\text { - Inpatient teledermatology during the } \\
\text { COVID-19 pandemic }\end{array}$ & $\begin{array}{l}\text { Julio } \\
2020\end{array}$ & $\begin{array}{l}\text { Carta al } \\
\text { Editor }\end{array}$ & $\begin{array}{l}\text { Estados } \\
\text { Unidos }\end{array}$ & Sí & $\begin{array}{l}\text { Rismiller et al. } \\
2020\end{array}$ \\
\hline $\begin{array}{l}\text { Not the pandemic but telemedicine } \\
\text { will prevent dermatology patients from } \\
\text { overloading the healthcare system }\end{array}$ & $\begin{array}{c}\text { Septiembre } \\
2020\end{array}$ & $\begin{array}{l}\text { Artículo } \\
\text { breve }\end{array}$ & Turquía & No & $\begin{array}{l}\text { Adısen et al. } \\
2020\end{array}$ \\
\hline $\begin{array}{l}\text { Telemedicine for inpatient } \\
\text { dermatology consultations in } \\
\text { response to the COVID-19 pandemic }\end{array}$ & $\begin{array}{l}\text { Julio } \\
2020\end{array}$ & $\begin{array}{l}\text { Carta al } \\
\text { editor }\end{array}$ & $\begin{array}{l}\text { Estados } \\
\text { Unidos }\end{array}$ & Sí & $\begin{array}{c}\text { Trinidad et al. } \\
2020\end{array}$ \\
\hline
\end{tabular}


manera similar, otro de los artículos mencionó que sólo un $4.8 \%$ del total de sus teleconsultas ameritó una visita presencial. ${ }^{16,17}$

\section{DISCUSIÓN}

El rol de la telemedicina ha sido controvertido desde hace ya varios años, pues se menciona que daña la relación médico-paciente pues reduce la dignidad humana. ${ }^{13} \mathrm{Si}$ bien hay retos en dicha relación que deben abordarse, la telemedicina es capaz de incluir lo mejor de ambos mundos, una práctica más disponible sin perder el factor humano. ${ }^{18}$ Y continuará siendo útil aún después de la pandemia. ${ }^{19}$

Entre sus principales beneficios durante la pandemia se encuentra la disminución de exposición a riesgo de los pacientes, lo cual es de vital importancia para aquéllos con factores de riesgo para la enfermedad como lo son el tratamiento inmunomodulador en algunos padecimientos dermatológicos y reumáticos, y pacientes de edad avanzada; en especial en padecimientos que no ameritan procedimientos quirúrgicos. ${ }^{16,20}$

Dentro de los modelos y algoritmos propuestos por algunos autores, destaca la importancia de un sistema de triaje que permita a los profesionales de la salud identificar de forma correcta aquellos padecimientos de importancia médica como el melanoma. ${ }^{20}$ Este tipo de modelos de atención contribuyen a incrementar el costo beneficio de los programas de teledermatología, en especial al utilizarse en asociación con otras herramientas digitales, como el expediente clínico virtual. ${ }^{21}$ Es importante destacar que se ha reportado el uso de plataformas de uso libre o de menor costo como Zoom en la consulta dermatológica, buscando reducir los costos presentados por los centros de salud al adherirse a este enfoque. ${ }^{22,23}$ Otro de los beneficios incluyen la reducción en uso de equipo de protección personal (EPP), el cual podrá destinarse a los médicos de primer contacto, pues a menor flujo de pacientes en las clínicas podrá redirigirse de mejor manera el insumo. ${ }^{21,24}$

Al momento, los estudios realizados en el campo de la dermatología han mostrado que una cantidad significativa de los padecimientos que acudieron a consulta podrían haber sido tratados por medio de telemedicina, pues las principales causas de consulta incluyen acné y dermatitis. ${ }^{16,17}$ Sin embargo, otras investigaciones muestran que las consultas actuales en su experiencia no representan ni el $50 \%$ del total de las consultas en físico previo a la pandemia. ${ }^{15}$

Por otro lado, también cuenta son sus limitantes, una de las principales es la incertidumbre legal en la que se rige la teledermatología; en países desarrollados como España se encuentran ya con normas bien establecidas para la regulación de esta práctica. ${ }^{11}$ Otros, en vías de desarrollo, aún no han implementado los marcos legales necesarios, vulnerando a los profesionistas de la salud y sus pacientes. En este sentido, la ética recomendada se mantiene adherida a los pilares fundamentales de la práctica médica, Autonomía, Beneficencia, Justicia y No maleficencia buscando no disminuir la satisfacción de los pacientes. ${ }^{17}$

Otra limitación relevante que se presenta con más frecuencia en países en vías de desarrollo se relaciona con la falta de condiciones para la realización de la consulta, desde equipo de cómputo hasta falta de internet en algunas regiones que limitan la calidad de la atención. ${ }^{25}$ Por lo que su impacto en países en vías de desarrollo o comunidades rurales se ve afectado.

Por último, se sugiere una serie de puntos de buena práctica en algunos de los artículos revisados, iniciando por el uso del consentimiento informado y el respeto a la autonomía del paciente en su decisión sobre el formato de la consulta. ${ }^{21}$ Además del uso de distintas herramientas tecnológicas para complementar las barreras en la infraestructura, tales como el uso de fotografía de las lesiones y la posibilidad de equipos de video que permitan la apreciación desde distintos ángulos, ${ }^{26}$ igualmente se considera de importancia la necesidad de educar a los pacientes en signos de alarma o en gravedad de las enfermedades, pues en muchas ocasiones lo que es urgente para el médico no lo es para el paciente y viceversa; además de instruirlos acerca de la forma correcta de tomar imágenes.

Esta pandemia representa un reto sin precedentes para la práctica médica y, si bien la telemedicina no puede resolver todas las problemáticas, sí se vuelve una herramienta única, en especial para especialidades como la dermatología que dependen por completo de la exploración de las lesiones. ${ }^{27}$ Si bien la práctica puede representar un reto para algunos profesionistas regidos con ética y confidencialidad, podemos llegar a dar una atención de calidad con el factor humano. Considerando la situación actual y a corto plazo, este tipo de prácticas se vuelve nuestra mejor oportunidad para evitar riesgos innecesarios y disminuir la carga del sistema de salud.

\section{REFERENCIAS}

1. Hurst EJ. Evolutions in telemedicine: from smoke signals to mobile health solutions. J Hosp Librariansh. 2016; 16 (2): 174-185. Available from: http://www.tandfonline.com/doi/full/10.1080/15323269.201 6.1150750

2. National Library of Medicine. (1993). Telemedicine. https://www. ncbi.nlm.nih.gov/mesh?Db=mesh\&Cmd=DetailsSearch\&Term $=\%$ 22Telemedicine $\% 22 \% 5 \mathrm{BMeSH}+$ Terms\%5D

3. Moore M. The evolution of telemedicine. Futur Gener Comput Syst. 1999; 15 (2): 245-254. Available from: https://linkinghub.elsevier. com/retrieve/pii/S0167739X98000673

4. Jin Z, Chen Y. Telemedicine in the cloud era: prospects and challenges. IEEE Pervasive Comput. 2015; 14 (1): 54-61. Available from: http:// ieeexplore.ieee.org/document/7030248/ 
5. Elliott T, Yopes MC. Direct-to-consumer telemedicine. J Allergy Clin Immunol Pract. 2019; 7 (8): 2546-2452. Available from: https:// linkinghub.elsevier.com/retrieve/pii/S2213219819305999

6. Jain T, Lu RJ, Mehrotra A. Prescriptions on demand: the growth of direct-toconsumer telemedicine companies. JAMA. 2019; 322 (10): 925. Available from: https://jamanetwork.com/journals/jama/fullarticle/2740743

7. Hatcher-Martin JM, Adams JL, Anderson ER, Bove R, Burrus TM, Chehrenama $M$ et al. Telemedicine in neurology: telemedicine work group of the American Academy of Neurology update. Neurology. 2020; 94 (1): 30-38. Available from: http://www.neurology.org/ lookup/doi/10.1212/WNL.0000000000008708

8. Dahy A, El-Qushayri AE, Mahmoud AR, Al-kelany TA, Salman S. Telemedicine approach for psoriasis management, time for application? A systematic review of published studies. Dermatol Thert. 2020; Available from: https://onlinelibrary.wiley.com/doi/ abs/10.1111/dth.13908

9. Makhni MC, Riew GJ, Sumathipala MG. Telemedicine in orthopaedic surgery. J Bone Jt Surg [Internet]. 2020; Publish Ah. Available from: https://journals.Iww.com/10.2106/JBJS.20.00452

10. Iancu AM, Kemp MT, Alam HB. Unmuting medical students' education: utilizing telemedicine during the COVID-19 pandemic and beyond. J Med Internet Res. 2020; 22 (7): e19667. Available from: http://www.jmir.org/2020/7/e19667/

11. Arimany-Manso J, Pujol RM, García-Patos V, Saigí U, MartinFumadó C. Aspectos médico-legales de la teledermatología. Actas Dermosifiliogr. 2020; Available from: https://linkinghub.elsevier.com/ retrieve/pii/S0001731020303136

12. Mehrotra A, Huskamp HA, Souza J, Uscher-Pines L, Rose S, Landon $\mathrm{BE}$, et al. Rapid growth in mental health telemedicine use among rural medicare beneficiaries, wide variation across states. Health Aff. 2017; 36 (5): 909-917. Available from: http://www.healthaffairs.org/ doi/10.1377/hlthaff.2016.1461

13. Menage J. Why telemedicine diminishes the doctor-patient relationship. BMJ. 2020; m4348. Available from: https://www.bmj. com/lookup/doi/10.1136/bmj.m4348

14. Mann DM, Chen J, Chunara R, Testa PA, Nov O. COVID-19 transforms health care through telemedicine: evidence from the field. J Am Med Informatics Assoc. 2020; 27 (7): 1132-1135. Available from: https:// academic.oup.com/jamia/article/27/7/1132/5824298

15. Perkins S, Cohen JM, Nelson CA, Bunick CG. Teledermatology in the era of COVID-19: experience of an academic department of dermatology. J Am Acad Dermatol. 2020; 83 (1): e43-44. Available from: https://linkinghub.elsevier.com/retrieve/pii/ S0190962220306617

16. Adisen E, Afacan E, Gürer MA. Not the pandemic but telemedicine will prevent dermatology patients from overloading the healthcare system. Dermatol Ther [Internet]. 2020; 1-5. Available from: https:// onlinelibrary.wiley.com/doi/10.1111/dth.14302
17. Kazi R, Evankovich MR, Liu R, Liu A, Moorhead A, Ferris LK et al. Utilization of asynchronous and synchronous teledermatology in a large health care system during the COVID-19 pandemic. Telemed e-Health. 2020. Available from: https://www.liebertpub.com/ doi/10.1089/tmj.2020.0299

18. Luz PL da. Telemedicine and the doctor/patient relationship. Arq Bras Cardiol. 2019; 113 (1). Available from: https://www.scielo.br/scielo. php?script=sci_arttext\&pid $=$ S0066-782X2019000700100

19. Stokel-Walker C. Why telemedicine is here to stay. BMJ. 2020; m3603. Available from: https://www.bmj.com/lookup/doi/10.1136/ bmj.m3603

20. Gomolin T, Cline A, Handler MZ. The danger of neglecting melanoma during the COVID-19 pandemic. J Dermatolog Treat. 2020; 31 (5): 444-445. Available from: https://www.tandfonline.com/doi/full/10. 1080/09546634.2020.1762844

21. Rismiller K, Cartron AM, Trinidad JCL. Inpatient teledermatology during the COVID-19 pandemic. J Dermatolog Treat. 2020; 31 (5): 441-443. Available from: https://www.tandfonline.com/doi/full/10. 1080/09546634.2020.1762843

22. Trinidad J, Kroshinsky D, Kaffenberger BH, Rojek NW. Telemedicine for inpatient dermatology consultations in response to the COVID-19 pandemic. J Am Acad Dermatol. 2020; 83 (1): e69-71. Available from: https://doi.org/10.1016/j.jaad.2020.04.096

23. Beer J, Abrouk M, Kirsner R. Telemedicine platforms used in academic dermatology during the COVID-19 pandemic: implications for adaptation and usage. J Drugs Dermatology. 2020; 19 (8): 797798. Available from: https://jddonline.com/articles/dermatology/ S1545961620P0797X

24. Hammond MI, Sharma TR, Cooper KD, Beveridge MG. Conducting inpatient dermatology consultations and maintaining resident education in the COVID-19 telemedicine era. J Am Acad Dermatol. 2020; 83 (4): e317-318. Available from: https://doi.org/10.1016/j. jaad.2020.07.008

25. Kumar S, Bishnoi A, Vinay K. Changing paradigms of dermatology practice in developing nations in the shadow of COVID-19: lessons learnt from the pandemic. Dermatol Ther. 2020; 33 (4): 3-5. Available from: https://onlinelibrary.wiley.com/doi/10.1111/dth.13472

26. Abi Rafeh J, Cattelan L, Zargham H, Jafarian F. Growing role of telemedicine in dermatology: a practical, timely application for skin cancer screening in organ transplant recipients. J Cutan Med Surg. 2020; 120347542095762. Available from: https://doi. org/10.1177/1203475420957626

27. Hollander JE, Carr BG. Virtually Perfect? Telemedicine for Covid-19. N Engl J Med. 2020; 382 (18): 1679-1681. Available from: http:// www.nejm.org/doi/10.1056/NEJMp2003539

Conflicto de intereses: Sin conflicto de intereses. 